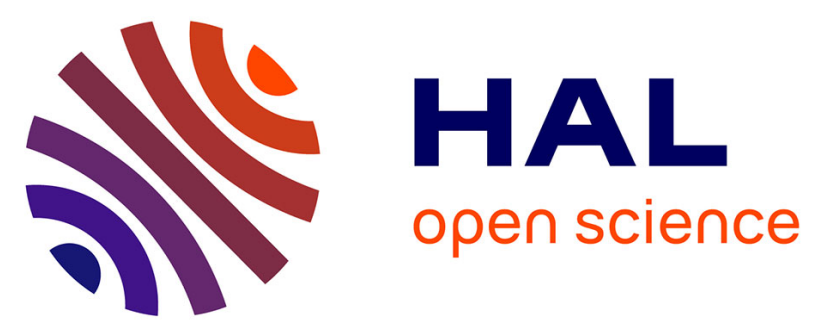

\title{
An Effective Location-Based Alert Messages Dissemination Scheme for Software Defined Vehicular Networks
}

\author{
Raoua Chakroun, Thierry Villemur, Slim Abdellatif
}

\section{- To cite this version:}

Raoua Chakroun, Thierry Villemur, Slim Abdellatif. An Effective Location-Based Alert Messages Dissemination Scheme for Software Defined Vehicular Networks. 35th International Conference on Advanced Information Networking and Applications (AINA-2021), May 2021, Toronto, Canada. pp.542552, 10.1007/978-3-030-75100-5_47. hal-03561875

\section{HAL Id: hal-03561875 \\ https://hal.science/hal-03561875}

Submitted on 17 Feb 2022

HAL is a multi-disciplinary open access archive for the deposit and dissemination of scientific research documents, whether they are published or not. The documents may come from teaching and research institutions in France or abroad, or from public or private research centers.
L'archive ouverte pluridisciplinaire HAL, est destinée au dépôt et à la diffusion de documents scientifiques de niveau recherche, publiés ou non, émanant des établissements d'enseignement et de recherche français ou étrangers, des laboratoires publics ou privés. 
See discussions, stats, and author profiles for this publication at: https://www.researchgate.net/publication/351086606

\section{An Effective Location-Based Alert Messages Dissemination Scheme for Software Defined Vehicular Networks}

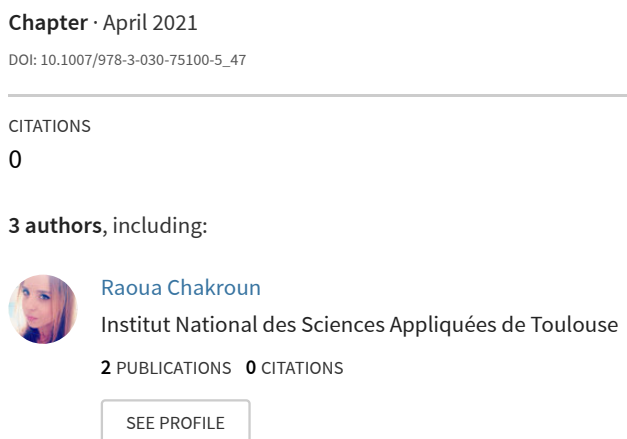

READS

49

Thierry Villemur

Laboratoire d'Analyse et d'Architecture des Systèmes (LAAS)

62 PUBLICATIONS 270 CITATIONS

SEE PROFILE 


\title{
An Effective Location-based Alert Messages Dissemination Scheme For Software Defined Vehicular Networks
}

\author{
Raoua Chakroun*, Slim Abdellatif* and Thierry Villemur*
}

\begin{abstract}
Software Defined Networking (SDN) is considered as a key paradigm for future vehicular networks and its adoption may pave the way to novel approaches to support emerging and legacy ITS services, such as Alert Message (AM) dissemination. Historically, to meet the low dissemination delay requirements and full vehicle coverage, Vehicular Ad-hoc NeTworks (VANETs) were considered as the underlying assumption of most proposed AM dissemination schemes. With SDN, vehicularto-infrastructure (V2I) links are a viable alternative that adds to Vehicle-to-vehicle (V2V) links. Moreover, a global view of the network at a centralized controller complemented with information on vehicles and their route helps to adjust the dissemination procedure to improve its performance and efficiency. In this paper, we propose a location-based AMs dissemination scheme that combines V2I broadcasts with V2V rebroadcasts in order to provide a low delivery delay, very limited collisions, high information coverage with insignificant signaling and network overhead. The originality of our scheme stands in the selection process of $\mathrm{V} 2 \mathrm{~V}$ rebroadcasts which is based on vehicles' location with respect to predefined rebroadcast points selected by the controller. Our proposal is evaluated and compared to legacy techniques. It shows significant improvements in delivery delays and network resource utilization.
\end{abstract}

\section{Introduction}

As an obvious evolution of legacy ITS (Intelligent Transportation Systems), Cooperative ITS (C-ITS) have been pushed in the front of the stage with the idea of enabling communications between vehicles (V2V: Vehicle-to-Vehicle), vehicles and infrastructure (V2I: Vehicle-to-Infrastructure), and more generally vehicles and all their surroundings (V2X: Vehicles-to-everything). The rationale is that thanks to

* LAAS-CNRS, 7 avenue du colonel roche, Toulouse, France e-mail: \{rchakrou,slim,villemur\}@laas.fr 
these new communication alternatives, the perception of vehicles on their surrounding and their environment is enriched, its accuracy is improved and the perception horizon is enlarged. As a consequence, novel C-ITS services that further improve road safety, traffic efficiency, comfort and convenience to drivers and passengers are envisioned, e.g. cooperative maneuver, bird's eye view, cooperative awareness (e.g. Emergency vehicle warning, Alert for an accident), etc. [15, 11].

One of the challenges facing the development of C-ITS is the provision of network connectivity services that meet the various Quality of Service (QoS) expectations of C-ITS services. One direction that has adhesion from academia and Industry is to consider a hybrid multi-access vehicular network that combines, amongst: DSRC (Dedicated Short Range Communication) which is foreseen for rapid dissemination and delivery of critical safety messages, and LTE(Long Term Evolution) which is foreseen for heavier and less stringent message exchanges [20, 15]. One promising approach towards this hybridization is to apply the Software Defined Networking (SDN) paradigm as leverage to unify the network control of the abovecited technologies with lots of promising opportunities and outcomes in terms of improved network resource usage and performance [7, 9, 16]. For these reasons, this work considers SDN-based vehicular networks.

Point-to-multi-point communications and more specifically Geo-Broadcasting are at the heart of many C-ITS services. Alert Message AM dissemination is such a service, which upon an emergency or a risky situation (accident or vehicle breakdown), alert messages are generated for some time and spread over a geographical area of interest as fast as possible to allow other vehicles to react properly very quickly [3]. Historically, message alert dissemination was carried out by a DSRC based VANET (Vehicular Ad-hoc NeTworks) operating on a dedicated communication channel by inviting vehicles to blindly rebroadcast the alerts. This method, known as simple flooding, causes a huge amount of unnecessary re-transmissions and collisions (known as the broadcast storm problem), thus wasting bandwidth, increasing dissemination delay, and lowering packet delivery ratio [17]. Lots of proposals from the literature tried to mitigate the broadcast storm problem by controlling the vehicles that are allowed to rebroadcast (using some form of random selection or clustering techniques to group vehicles) or reducing the contention. But, most were designed for VANETs and neither assume the presence of V2I links nor take advantage of the benefits that a global view of the network at a centralized controller can bring to the dissemination scheme.

In this paper, we propose an alert messages dissemination scheme that primarily exploits V2I transmissions and complements with V2V transmissions in order to reach vehicles located in areas that are not covered (white zones) or poorly covered (grey zones) by transmissions from the infrastructure. The originality of our scheme stands in the selection process of relay vehicles which is based on a local decision at each vehicle based on its location with respect to predefined rebroadcast points that are computed by the controller based on its knowledge of the radio performance and coverage as well as road traffic conditions. In addition, our proposal induces marginal extra signaling and network overhead. 
The rest of the paper is organized as follows: Section 2 presents an overview of existing work on AM dissemination. Section 3 describes the system model as well as the proposed location-based dissemination algorithm. Section 4 evaluates and discusses our simulation results. Finally, Section 5 concludes the paper.

\section{Related Work}

Alert message dissemination has been widely studied in a VANET/V2V context. All aim at mitigating the effect of the "broadcast storm" while ensuring high information coverage. These works can be broadly classified as follows.

Lots of the proposed schemes try to control the flooding procedure of AMs either by reducing the number of rebroadcasters or by reducing the contention between transmitting vehicles. In the former case, some vehicles are selected to relay the AMs $[14,2,19]$. This selection is based on vehicles' characteristics, distance from the sender, local density, interests, transmit power, etc., and helps to limit retransmissions and contention at the cost of reduced information coverage. In the latter case, MAC level protocol parameters are adjusted on a per vehicle basis in order to statistically assign different back-off periods to vehicles [6]. By so doing, collisions are reduced with no impact on information coverage. However, useless transmissions are not avoided.

Another class of proposals set aside the flooding logic and try to guide AMs dissemination by organizing vehicles in clusters (groups of vehicles) and defining how AM are propagated between and within clusters $[8,10,18]$. Many criteria are used for cluster formation as well as cluster-head selection, e.g. neighborhood, direction/destination, relative velocity, etc. A last class of proposals is those based on routing protocols that proactively or reactively compute point-to-multi-point routes to all known vehicles [4]. They clearly exhibit the most predictable information coverage and efficient resource usage, but this comes at the cost of increased network overhead and complexity embedded in vehicles.

\section{Location-based Alert Messages Dissemination}

\subsection{System Model}

An SDN controller is assigned to each region, it manages all the RSUs that fall inside. Each controller identifies whites and grey zones in its region and selects $K$ rebroadcast zones where vehicles can rebroadcast an AM sent by an RSU in order to enhance the overall coverage and reach all the vehicles located in grey or white zones. These rebroadcast zones are defined by a rebroadcast point $P_{i}\left(x_{i}, y_{i}\right)$, $i \in[1, K]$, with $x_{i}$ and $y_{i}$ are the GPS coordinates of $P_{i}$, and a radius $d_{\max }$ in the order 
Fig. 1 Rebroadcast zones

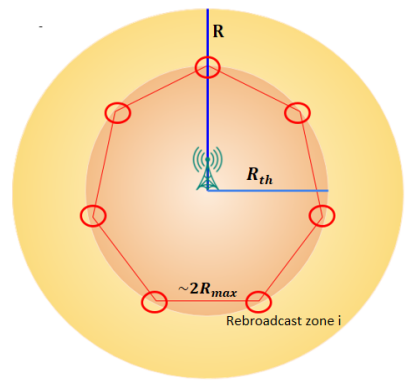

of a few meters from the rebroadcast point. The contribution of this paper concerns the dissemination procedure, as a consequence, a basic broadcast point placement is adopted as explained hereafter.

Real experiments in [20] show that the packet loss rate and delay increase when the distance between vehicles and RSU is greater than a threshold $R_{t h}$ which also depends on mobility, due to the wireless propagation channels. According to [15], an RSU will be deployed every $2 \mathrm{~km}$ in Europe with a theoretical coverage defined by $R=850 \mathrm{~m}$. We assume that the controller is able to define a threshold distance $R_{t h}$ for each RSU from which the quality degrades and the probability of reception decreases. From this threshold radius (Fig. 1), it can build a regular polygon with $K, K \in[5,17]$ equal sides of $2 R_{\max }( \pm 50 \mathrm{~m})$ (where $R_{\max }$ is the maximum radius coverage of a vehicle), to avoid collisions between two vehicles in two adjacent rebroadcast zones, whose polygon vertices represent the rebroadcast points. In this case, a rebroadcast zone $i$ is represented by the vertex $P_{i}$ with a margin $d_{\max }$ of a few meters. If a rebroadcast zone does not serve any road area, it will be omitted.

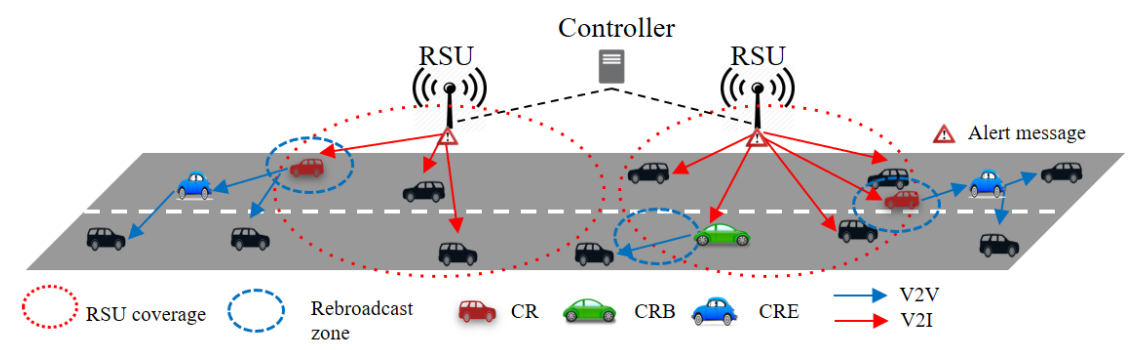

Fig. 2 Location-based alert messages dissemination 


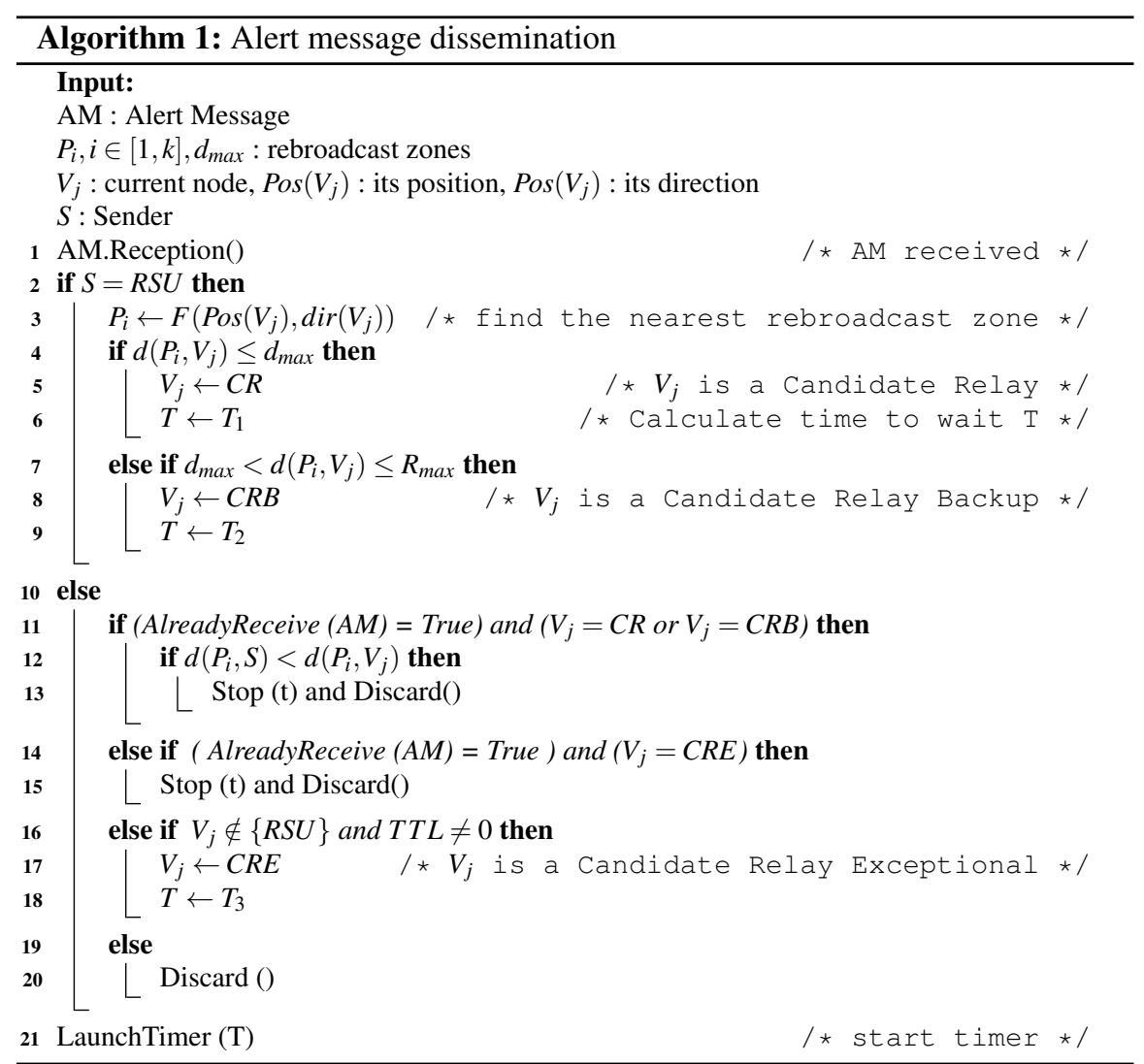

\subsection{Dissemination Procedure}

The proposed AM dissemination scheme is described in Algorithm 1. At handover, on the re-association request with a new RSU, a vehicle $V_{j}$ receives the list of the rebroadcast zones that are under the coverage of the RSU. An AM is broadcasted with the position of the sender and a predefined Time To Live TTL (used to control the rebroadcasts of vehicles located in white zones as shown in Fig. 2). Vehicles that receive an AM check their location: inside, around, or away from the rebroadcast zones. The vehicle closest to the broadcast point is the best candidate for the rebroadcast. This is achieved by assigning, on an AM reception, a personalized arbitration waiting time to each vehicle. The closer a vehicle is from the rebroadcast point, the lower is its waiting time.

Let $P_{i}=F\left(\operatorname{pos}\left(V_{j}\right), \operatorname{dir}\left(V_{j}\right)\right)$ a function which takes the GPS position of vehicle $\operatorname{pos}\left(V_{j}\right)=\left(x_{j}, y_{j}\right)$ and its direction as parameters, and returns the closest rebroadcast zone in its direction.

referring to Alg. 1 , if a vehicle $V_{j}$ receives an AM, first, it checks the sender: 
Step 1 [Lines 2-3] If the sender is an RSU, the node finds the closest rebroadcast point $P_{i}$ to its location, and it checks its position according to the rebroadcast zone, otherwise, the message is received from a relay vehicle, go to step 4

Step 2 [Lines 4-6] If $V_{j}$ is in the rebroadcast zone, the node is declared as Candidate Relay $\mathrm{CR}$ and the vehicle in the rebroadcast zone closest to the rebroadcast point has the highest priority to rebroadcast, so it has the minimum waiting time $T_{1}$. Another factor that we consider is the distance between the RSU (sender) and $V_{j}$ (receiver) to avoid the simultaneous transmissions of multiple vehicles sitting at the same distance from the rebroadcast point. Hence, a relaying metric $G_{1}$ that is used to calculate the time to wait $T_{1}$, is given by :

$$
G_{1}=(1-\alpha) \frac{d\left(V_{j}, S\right)}{R}+\alpha\left(1-\frac{d\left(V_{j}, P_{i}\right)}{d_{\max }}\right)
$$

where : $d\left(V_{j}, S\right)$ indicates the distance between vehicle $V_{j}$ and RSU, $R$ is maximum transmission coverage of the RSU, $d\left(V_{j}, P_{i}\right)$ is the distance between vehicle $V_{j}$ and rebroadcast point $P_{i}, d_{\max }$ is the radius of a rebroadcast zone. $\alpha$ determines the influence of each component( distance from the rebroadcast point and distance from the RSU) to compute $G_{1}$. Hence, if we consider a DSRC enabled vehicle, the waiting time $T_{1}$ is derived as follows:

$$
T_{1}=\left(C W_{\min }-G_{1} C W_{\min }\right) T_{s}
$$

where $C W_{\min }$ and $T_{s}$ are respectively the minimum contention window and the time slot duration of DSRC's medium access technique, namely CSMA/CA (Carrier Sense Multiple Access/Collision Avoidance) [1]. Go to step 7, otherwise, go to step 3

Step 3 [Lines 7-9] If $V_{j}$ is in the radius coverage $R_{\max }$ of rebroadcast point, then, the node is declared as Candidate Relay Backup CRB and the relaying metric $G_{2}$ is given by :

$$
G_{2}=(1-\alpha) \frac{d\left(V_{j}, S\right)}{R}+\alpha\left(1-\frac{d\left(V_{j}, P_{i}\right)}{R_{\max }}\right)
$$

Hence for a DSRC enabled vehicle, the waiting time $T_{2}$ is as follows:

$$
T_{2}=\left[C W_{\min }+\left[\left(C W_{\max }-C W_{\min }\right)-G_{2}\left(C W_{\max }-C W_{\min }\right)\right]\right] T_{S}
$$

where $C W_{\max }$ is the maximum contention window related to unicast transmissions. $T_{2}$ is set between $C W_{\min }$ and $C W_{\max }$ to avoid collisions with nearby CR vehicles. This is particularly useful when the road traffic is low or sparse since, in case of no vehicle in a broadcast zone, a close-by vehicle is given the opportunity to rebroadcast the AM. Go to step 8, Otherwise discard the message 
Step 4 [Lines 10-11] If $V_{j}$ receives the same message another time, and $V_{j}$ is a CR or a CRB go to step 5, otherwise, go to step 6

Step 5 [Lines 12-13] If $V_{j}$ and the sender node are in the same rebroadcast zone, i.e. the distance between the rebroadcast point and the sender is less than the distance between the rebroadcast point and $V_{j}$, stop the timer, the procedure exits, otherwise, go to Step 9

This is to allow only the closest vehicle to the rebroadcast point to rebroadcast (if both vehicles are in the same rebroadcast zone). It also avoids that a CR or CRB related to a rebroadcast point to be prevented from rebroadcasting when it receives a rebroadcast AM related to another rebroadcast point.

Step 6 [Lines 14-15] If $V_{j}$ receives the same message another time, and $V_{j}$ is a Candidate Relay Exceptional CRE, stop the timer, the procedure exits, otherwise, go to Step 7

Step 7 [Lines 16-18] If $V_{j}$ is not attached to any RSU and the TTL is not expired, $V_{j}$ is declared as CRE. In this case, the farthest vehicle from the sender, but still in its transmission range, has the highest priority to rebroadcast, so the relaying metric $G_{3}$ is given by :

$$
G_{3}=\frac{d\left(V_{j}, S\right)}{R_{\max }}
$$

Hence the waiting time $T_{3}$ is calculated as follows:

$$
T_{3}=\left(C W_{\min }-G_{3} C W_{\min }\right) T_{S}
$$

Otherwise, Discard message

Step 8 [Line 21] Start back-off Timer $T$

Step 9 Having waited for the time $T, V_{j}$ decrements $T T L$ and forwards the message.

\section{Performance Evaluation}

The objective is to assess the performance of our method: (1) to reach all the vehicles sitting in the area of interest in a very short time,(2) to avoid collisions, and (3) to effectively use network resources. Also, our proposal is compared to the flooding technique [5] and V2I broadcasts (with no V2V rebroadcasts). The following performance metrics are used:

Collision Ratio: The collision ratio is the percentage of MAC collisions divided by the number of sent packets.

Information Coverage: computed as the total number of vehicles that successfully receive the AM divided by the number of all the vehicles. 
Packet Delivery Ratio: is the ratio between the number of successfully received packets at the vehicles of the area of interest and all transmitted packets

Dissemination delay: The dissemination delay is the total time required to deliver the AM to all the vehicles in the area of interest.

\subsection{Simulation Setup}

In the experiments, an event based network simulator Netsim was used. In order to make the most realistic simulations, SUMO (Simulation of Urban Mobility) bidirectionally coupled with Netsim as described in [12] was used. Netsim includes implementations of IEEE 1609.4 and IEEE 802.11p communication standards. It further includes Basic Safety Message (BSM) handling and beaconing for cooperative awareness messages (CAMs). In order to create realistic road traffic scenarios on a real map with SUMO, we used OpenStreetMap as the geographic data source and selected an area of $2 \mathrm{~km} \times 2 \mathrm{~km}$ located in the city center of Toulouse, France. The traffic of vehicles was generated randomly. We inserted vehicles into the network topology at a constant rate $(30,75,100,200,300,400$, and 500 vehicles). The speed of vehicles was varied from 0 to $20 \mathrm{~m} / \mathrm{s}$, i.e. vehicles had different accelerations at different timestamps. The maximum transmission range of each vehicle was set to $R_{\max }=250 \mathrm{~m}$. In all simulated scenarios, there was two RSUs separated by a distance of $2 \mathrm{~km}$. Each one was responsible for the transmission of alert messages to vehicles located in its transmission range. The other simulation parameters are shown in Table 1.

\subsection{Rebroadcast Zone Selection}

For the performance evaluation of our dissemination procedure, we considered a basic and empirical rebroadcast points/zones selection method described hereafter. The goal is to compute a threshold distance $R_{t h}$ associated to the two considered RSUs and build our polygon from which we derive the locations of rebroadcast points. To that end, each RSU broadcasts a control message every $100 \mathrm{~ms}$ for 500s. After each packet, we logged the distance of each vehicle from the RSUs, the vehicles that received the message, and the packet status (success or error). From the performance analysis, we observed that the packet loss rate dramatically increases when the distance between the vehicle and RSU is strictly greater than $R_{t h} \simeq 600 \mathrm{~m}$. From this threshold, we built a regular octagon for each RSU with 8 sides of $459 m\left(0.765 R_{t h}[13]\right)$ length and 8 vertices which represent our rebroadcast points. We did not place all the rebroadcast points as the RSUs are in the corners; we had a total of 9 rebroadcasts zones shown in Fig. 3. 


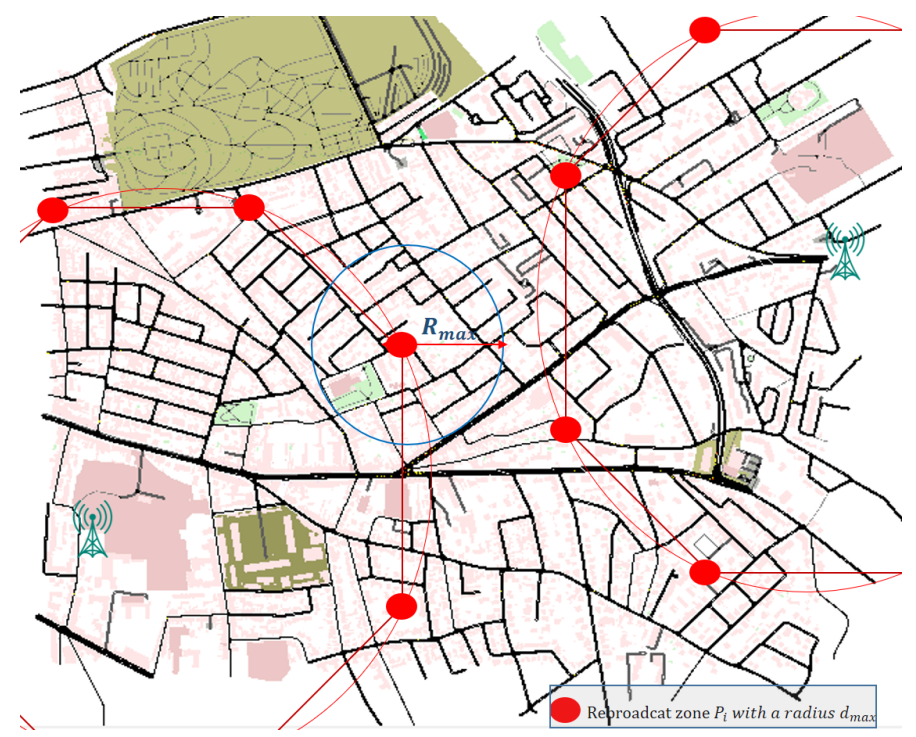

Fig. 3 Toulouse's city scenario and rebroadcast zones selection

Table 1 Simulation configuration parameters

\begin{tabular}{ll}
\hline Parameter & Value \\
\hline Simulation time & $500 \mathrm{~s}$ \\
Alert start & $10 \mathrm{~s}$ \\
Packet generation rate & 10 packets $/ \mathrm{s}$ \\
AM packet size & 1460 bytes \\
Propagation model & Nakagami $\mathrm{m}=3$ \\
Slot time & $16 \mu \mathrm{s}$ \\
$C W_{\min }$ & 15 \\
$C W_{\max }$ & 1023 \\
$d_{\max }$ & $16 \mathrm{~m}$ \\
$\alpha$ & 0.8 \\
\hline
\end{tabular}

\subsection{Performance Analysis}

Collision ratio Fig. 4 shows the collision ratio as a function of vehicle density. Even for high traffic density, we observe that, compared to flooding, our method significantly reduces packet collisions (around $0.001 \%$ ). This is due to the fact that only vehicles around rebroadcast zones are allowed to relay the message with different waiting times for each vehicle. The occurrence of some packet collisions is due to the behavior of the CSMA/CA mechanism. If the channel is busy, relay nodes must wait for a random back-off time before re-transmission. So when two vehicles are very close to each other, they have almost a similar waiting time, thus, they may rebroadcast at the same time if the channel is busy.. 
Information Coverage Fig. 5 shows the percentage of information coverage versus vehicle density. For low traffic density, the percentage of information coverage of the proposed technique is close to other techniques, but it is still better. This is due to the fact that, in such a scenario, the presence of vehicles around all rebroadcast zones at each AM transmission is not guaranteed. Indeed, vehicles can join and leave the network quickly according to the random mobility of SUMO without receiving any message. When increasing traffic density, flooding is a bad option as shown in the figure. Our method outperforms flooding and V2I approaches, and in contrast to these latter, the information coverage strictly increases as more vehicles are covering the area of interest. This means that rebroadcasts are taking place without causing collisions.

Dissemination Delay Fig. 6 shows the dissemination delay versus vehicle density. The results show that the dissemination delay of our technique is significantly lower than those of the other techniques, especially in high traffic density. Indeed, at each message period, there is a relay vehicle in or around the rebroadcast zone to transfer quickly the message to other vehicles outside the RSUs coverage. For an urban area, the frequent collisions inherent to the flooding technique cause an additional delay, and the mono-broadcast V2I technique takes a longer time to reach the maximum number of vehicles in the area, as the vehicle must be in the coverage of an RSU to receive the message. In low density, fewer vehicles are available in the network, so the presence of vehicles around each rebroadcast zone from the first AM message is not guaranteed, thus, our technique takes more time to reach all vehicles, e.g. for vehicles density of 30 vehicles, the total time required is $518.8 \mathrm{~ms}$. Indeed, the average speed of vehicles is between 7 and $13 \mathrm{~m} / \mathrm{s}$, the vehicle can only move approximately $1 \mathrm{~m}$ in $100 \mathrm{~ms}$, therefore vehicles take more time to draw near the rebroadcast zones. It is worth noting that, in this low density scenario, more than $75 \%$ of vehicles have the message delivered within $100 \mathrm{~ms}$.

Packet delivery ratio Fig. 7 shows the packet delivery ratio versus vehicle density. The results show that the packet delivery ratio of our proposal is slightly better than the V2I technique. This is due to the fact that relay vehicles rebroadcast only reach their neighbors. Because of the blind re-transmission which may cause a collision or a useless transmission, flooding suffers from poor packet delivery ratios.

\section{Conclusion And Future Works}

In this paper, we propose an alert message dissemination scheme based on vehicle locations to provide high coverage with fast delivery. The main peculiarities of our solution are: First, the combination of V2I transmissions, where RSUs broadcast alert messages and $\mathrm{V} 2 \mathrm{~V}$ re-transmissions, where some selected relay vehicles rebroadcast the messages after a personalized waiting time, Second, the definition of broadcast zones by the SDN controller where relay vehicles are allowed to rebroadcast the message to reach all vehicles in the area of interest and notably those located 

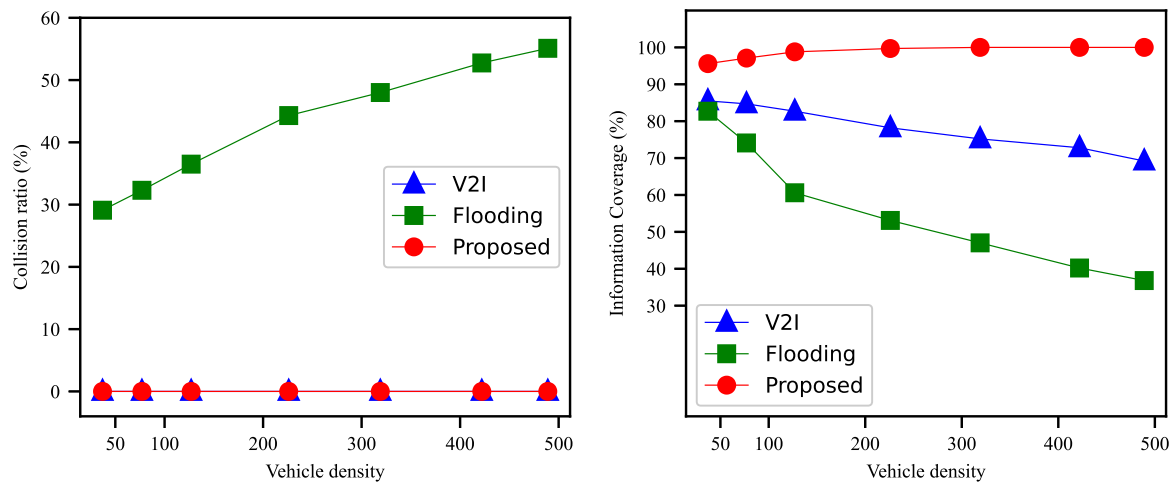

Fig. 4 Collision ratio vs. vehicle density

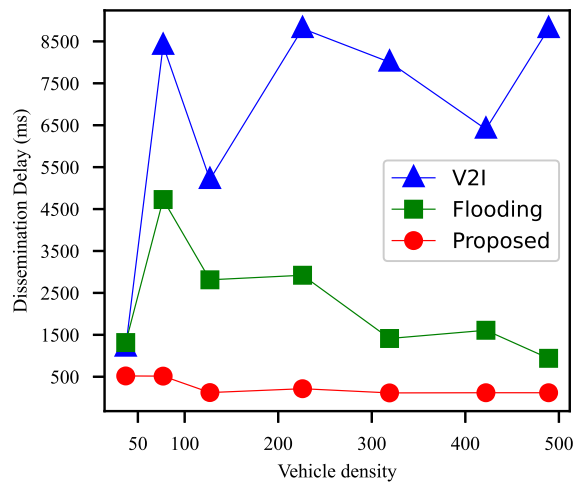

Fig. 6 Dissemination delay vs. vehicle density
Fig. 5 Information coverage vs. vehicle density

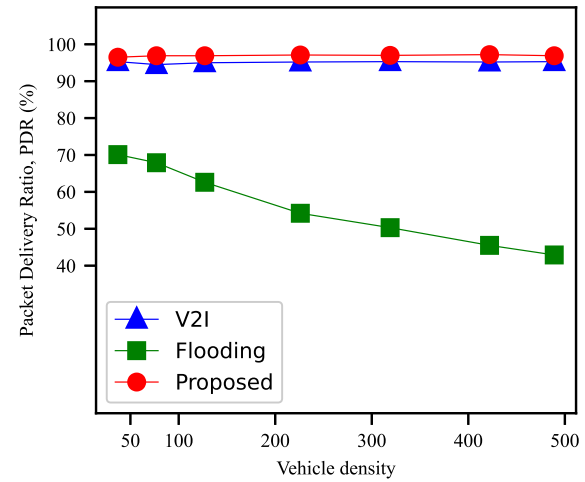

Fig. 7 Packet delivery ratio vs. vehicle density

in white or poorly covered areas. In this paper, we have proposed a static and simple method to define rebroadcast zones. The results obtained from our study prove the effectiveness of our scheme by maintaining its high delivery ratio, avoiding packet collisions, and ensuring rapid dissemination and high information coverage.

The main perspective of this work is to propose a machine-learning-based dynamic placement method of rebroadcast zones, which adapts to traffic conditions, weather, signal quality, etc.

Acknowledgements This work is funded by Continental Digital Service France (CDSF) in the framework of the eHorizon project. 


\section{References}

1. A. Ahizoune, A protocol for broadcasting messages in vehicular networks. thesis manuscript, 2011.

2. B. Liu, D. Jia, J. Wang, K. Lu, Cloud-assisted safety message dissemination in VANETcellular heterogeneous wireless network. IEEE Systems Journal, 2017. Vol. 11, p. 128-139.

3. F. D. Salim, S.W. Loke, A. Rakotonirainy, B. Srinivasan et S. Krishnaswamy. Collision pattern modeling and real-time collision detection at road intersections. Intelligent Transportation Systems Conference (ITSC),2007. p.161-166.

4. G. Aldabbagh, M. Rehan, H. Hasbullah, W. Rehan, and O. Chughtai, A driver safety information broadcast protocol for VANET. Appl. Math. Inf. Sci., 2016. vol. 10, p. 451-468.

5. G. Ciccarese, M. D. Blasi, P. Marra,On the use of control packets for intelligent flooding in VANETs. IEEE wireless communications and networking conference (WCNC 2009), Budapest, 2009. p. 1-6.

6. K. Virdaus, M. Kang, S. Shin, C. G. Lee, J. Pyun, A counting-based broadcast model of emergency message dissemination in VANETs. International Conference on Ubiquitous and Future Networks (ICUFN), Milan, 2017. p. 927-930.

7. K. Zheng, L. Kan, H. HOU, Q. Lu, N. MENG, L. Hanlin, Soft-defined heterogeneous vehicular network: Architecture and challenges. IEEE Network, 2016, vol. 30, p. 72-80.

8. L. Liu, C. Chen, T. Qiu, M. Zhang, S. Li, B. Zhou, A data dissemination scheme based on clustering and probabilistic broadcasting in VANETs. Vehicular Communication, Vol. 13, 2018. p. 78-88.

9. M. A. Salahuddin, A. Al-Fuqaha, M. Guizani, Software-defined networking for rsu clouds in support of the internet of vehicles. IEEE Internet of Things journal, 2014, vol. 2, p. 133-144.

10. M. Ali, A. Malik, A. Rahman, S. Iqbal, M. Hamayun. Position-based emergency message dissemination for Internet of vehicles.Distributed Sensor Networks, 2019. Vol. 15.

11. M. Boban, A. Kousaridac, K. Manlakis, J. Eichinger, W. Xu Use Cases, Requirements, and Design Considerations for 5G V2X, IEEE Vehicular Technology Magazine, 2017.

12. Netsim simulator. https://www.tetcos.com/vanets.html

13. Polygon construction. https://debart.pagesperso-orange.fr/geoplan/polygoneregulier.mobile.html

14. R. Oliveira, C. Montez, A. Boukerche, M. S. Wangham, Reliable data dissemination protocol for VANET traffic safety applications. Ad Hoc Networks, 2017. Vol. 63, p. 30-44.

15. Report of the working group: communication technologies for cooperative ITS. https://www.ecologie.gouv.fr/sites/default/files/Rapport

16. S. Toufga, S. Abdellatif, P. Owezarki, T. Villemur, D. Relizani, Effective Prediction of V2I Link Lifetime and Vehicle's Next Cell for Software Defined Vehicular Networks: A Machine Learning Approach. IEEE Vehicular Networking Conference (VNC), Los Angeles, CA, United States, 2019.

17. S. Y. Ni, Y. C. Tseng, Y. S. Chen, and J. P. Sheu, The broadcast storm problem in a mobile ad hoc network. the Fifth Annual ACM/IEEE International Conference on Mobile Computing and Networking, 1999. p. 152-162.

18. V. Velmurugan, J. M. L. Manickan, A efficient and reliable communication to reduce broadcast storms in VANET protocol. Cluster Computing, 2019.

19. W. Libing, N. Lei, F. Jin, H. Yanxiang, L. Qin, W. Dan, An Efficient Multi-hop Broadcast Protocol for Emergency Messages Dissemination in VANETs. Chinese Journal of Electronics, 2017. Vol. 26.

20. Z. Xu, X. Li, X. Zhao, M. H. Zhang, Z. Wang, DSRC versus 4G-LTE for Connected Vehicle Applications: A Study on Field Experiments of Vehicular Communication Performance. Journal of Advanced Transportation, 2017. vol. 2017. 